\title{
DIFERENCIAS EN CALIDAD DE VIDA ASOCIADO \\ A LA APARIENCIA NASAL EN PACIENTES OPERADOS DE RINOPLASTIA COMPARADO CON GRUPO CONTROL. VALIDEZ DEL INSTRUMENTO RHINOPLASTY OUTCOME EVALUATION*
}

Drs. Stefan Danilla E. ${ }^{1}$, María Elsa Calderón G. ${ }^{1}$, Pedro Cuevas T. ${ }^{1}$, Cristian Erazo C. ${ }^{1}$, Susana Benítez S. ${ }^{1}$, Patricio Andrades C. ${ }^{1}$, Sergio Sepúlveda P. ${ }^{1}$, Rolando Schultz R. ${ }^{1}$, Als. José Ignacio Vergara O. ${ }^{2}$, Felipe Soto V. ${ }^{2}$, Alma Cruz F. ${ }^{2}$, Sofía Serra D. ${ }^{2}$, Paula Silva R. ${ }^{2}$

1 Departamento de Cirugía, Hospital Clínico de la Universidad de Chile.

2 Alumnos Facultad de Medicina Universidad de Chile.

Santiago, Chile.

Abstract

\section{Evaluation of the Rhinoplasty Outcome Evaluation among Chilean participants}

Background: The Rhinoplasty Outcome Evaluation (ROE) is an instrument to assess the cosmetic and functional aspects of the nose from the patient's perspective. Its construct validity in Chilean subjects has been published previously. Aim: To compare the ROE scores in different groups of participants. Material and Methods: The questionnaire was applied to patients waiting for a rhinoplasty, patients hospitalized for other causes and medical students. Results: The overall reliability of the scale was $84.8 \%$. Significant differences between groups in the total scores of four out of six items were observed. Conclusions: The Spanish version of the ROE is adequate to evaluate the satisfaction with nasal appearance and function among Chilean individuals.

Key words: Rhinoplasty Outcome Evaluation, nose, rhinoplasty.

\section{Resumen}

Objetivo: El Rhinoplasty Outcome Evaluation (ROE) es un instrumento específico para la evaluación cosmética y funcional de la nariz desde la perspectiva del paciente, desarrollado originalmente en inglés. El objetivo del presente estudio es comparar las distribuciones en distintas poblaciones. Material y Método: El instrumento validado en español chileno $R O E$ fue sometido a prueba para evaluar su validez de criterio comparando los resultados de la escala en pacientes programados que deseaban someterse a rinoplastía versus 2

*Recibido el 10 de junio de 2013 y aceptado para publicación el 4 de septiembre de 2013.

Los autores no refieren conflictos de interés.

Correspondencia: Dr. Stefan Danilla E. drstefandanilla@gmail.com 
grupos control: 1) Pacientes hospitalizados por otras causas y 2) Estudiantes de medicina. La validez de constructo ya ha sido publicada por los creadores de la escala. Para la comparación de variables se utilizaron las pruebas $t$ de Student, ANOVA, Wilcoxon, Kruskall Wallis, prueba exacta de Fisher o $\chi^{2}$ según correspondiera. Para todas las pruebas se utilizó un nivel alfa de 5\%. Resultados: Se aplicó la encuesta a 45 pacientes. La edad promedio de la muestra fue de 35,4 años. La confiabilidad de la escala fue de $84,78 \%$. Se observaron diferencias estadísticamente significativas en el puntaje total $(p=0,0047)$ en 4 de los 6 ítems. Conclusión: Los resultados preliminares de nuestro estudio sugieren que la versión en Español del Rhinoplasty Outcomes Evaluation es útil para evaluar la satisfacción con la apariencia nasal en chilenos.

Palabras clave: Rinoplastía, calidad de vida, ROE.

\section{Introducción}

La rinoplastía es una de las cirugías más frecuentemente realizadas por cirujanos plásticos y otorrinolaringólogos en la última década, según la Sociedad Americana de Cirugía Plástica ${ }^{1}$. El surgimiento de nuevas técnicas quirúrgicas ${ }^{2,3}$, insumos, desarrollo tecnológico ${ }^{4}$ e imagenológico ${ }^{5,6}$ ha permitido mejorar la selección de pacientes y entregar resultados quirúrgicos más confiables.

Sin embargo, la evaluación sistemática de los resultados en rinoplastía no se ha realizado de forma rutinaria según los estándares de la Medicina Basada en Evidencia (MBE), lo que se refleja en la falta de estudios de alto nivel de evidencia ${ }^{7-9}$. En el campo de la cirugía estética la evaluación de los resultados postoperatorios es un tema complejo, motivo por el cual se han debido desarrollar distintos instrumen$\operatorname{tos}^{7,8}$ que permitan medir en forma cuantitativa los resultados obtenidos ${ }^{9,10}$. Uno de ellos corresponde al Rhinoplasty Outcome Evaluation ${ }^{11}$, que consiste en una encuesta aplicada en el preoperatorio y en el postoperatorio, donde el paciente asigna puntajes a distintos aspectos estéticos y funcionales relacionados a la nariz. El instrumento fue desarrollado en inglés y validado por nuestro grupo en español ${ }^{12}$.

El objetivo del presente estudio es evaluar la validez de criterio del ROE, realizando una comparación del puntaje obtenido por los pacientes programados para operarse de rinoplastía con un grupo control y evaluar la diferencia del puntaje entre el pre y post operatorio de los pacientes operados de rinoplastía.

El instrumento aplicado es una herramienta simple, reproducible, fácil de entender y de aplicar tanto para el cirujano como para el paciente.

\section{Material y Método}

\section{Diseño}

Estudio observacional prospectivo realizado en el Hospital Clínico Universidad de Chile.

\section{Pacientes}

Se denominó "Grupo Activo" a pacientes ingresados para realizar una rinoplastía estética. Además se ensamblaron 2 grupos control, uno correspondiente a alumnos (as) de medicina que no deseaban realizarse una rinoplastía y otro a pacientes hospitalizados por una causa distinta a una rinoplastía y que no quisieran realizarse una rinoplastía estética.

\section{Mediciones}

La variable principal de estudio fue la satisfacción con la apariencia nasal medida por el instrumento ROE. La validez de criterio del instrumento se sometió a prueba en el presente estudio. La validez de constructo y propiedades psicométricas de la escala ya han sido publicadas por sus autores ${ }^{14}$. El instrumento corresponde a un cuestionario de 6 ítems (preguntas) ordenados como preguntas Likert, esto es una afirmación y una escala de 5 puntos (Totalmente de acuerdo, de acuerdo, indiferente, desacuerdo, muy en desacuerdo) en que el paciente selecciona la respuesta que más se asemeja a su parecer (Tabla 1). La escala puede tomar un puntaje de 6 a 3 puntos, donde 6 puntos es el peor escenario y 30 el mejor ${ }^{13}$.

\section{Estadística}

La diferencia de puntaje entre grupos se evaluó con la prueba de Kruskall Wallis, la diferencia entre el grupo de rinoplastía entre el pre y postoperatorio se evaluó con la prueba de Wilcoxon. Las variables continuas entre grupos se evaluaron con la prueba de ANOVA y la prueba de $\chi^{2}$ para las variables categóricas. La confiabilidad se midió con el Alfa de Cronbach.

Para todas las pruebas se utilizó un nivel alfa de $5 \%$, en el análisis de los datos se utilizó el software Stata $10.2{ }^{\circledR}$ (Stata Corp, TX, EEUU).

\section{Resultados}

Entre marzo y agosto de 2011 se reclutaron 45 pacientes. Las características de los distintos grupos estudiados: edad, escolaridad e Índice de Masa Corporal (IMC) se describen en la Tabla 2.

La confiabilidad de la escala basada en el Alfa de Cronbach fue del $84,78 \%$. 
La mediana de respuestas por ítem en el preoperatorio, fue menor en el grupo de pacientes que desean operarse en comparación con el grupo control. Lo cual es coherente y consistente con la finalidad de la escala. La mediana del puntaje, desglosada por ítem se presenta en la Tabla 3.

En el grupo de pacientes operados, el puntaje final de la escala mejoró en forma significativa $(p=0,0047)$ en 4 de los 6 ítems, al comparar el preoperatorio con el postoperatorio Tabla 4.

Tabla 1. Encuesta Rhinoplasty Outcome Evaluation traducida y validada al español

\begin{tabular}{|c|c|c|c|c|}
\hline & $\begin{array}{c}\text { Totalmente en } \begin{array}{c}\text { Parcialmente } \\
\text { desacuerdo }\end{array} \text { en desacuerdo }\end{array}$ & $\begin{array}{l}\text { Ni acuerdo ni } \\
\text { en desacuerdo }\end{array}$ & $\begin{array}{l}\text { Parcialmente } \\
\text { de acuerdo }\end{array}$ & $\begin{array}{l}\text { Totalmente } \\
\text { de acuerdo }\end{array}$ \\
\hline \multicolumn{5}{|l|}{$\begin{array}{l}\text { a) La apariencia de mi nariz es la mejor } \\
\text { que puedo imaginar }\end{array}$} \\
\hline \multicolumn{5}{|l|}{$\begin{array}{l}\text { b) Puedo respirar perfectamente por mi } \\
\text { nariz }\end{array}$} \\
\hline \multicolumn{5}{|l|}{$\begin{array}{l}\text { c) A mis amigos y familia les gusta mi } \\
\text { nariz }\end{array}$} \\
\hline \multicolumn{5}{|l|}{$\begin{array}{l}\text { d) Nunca me he sentido limitado en mis } \\
\text { actividades sociales-laborales por la } \\
\text { apariencia de mi nariz }\end{array}$} \\
\hline \multicolumn{5}{|l|}{$\begin{array}{l}\text { e) Estoy seguro que la apariencia de mi } \\
\text { nariz es la mejor que puedo tener }\end{array}$} \\
\hline $\begin{array}{l}\text { f) No me gustaría cambiar quirúrgica- } \\
\text { mente la apariencia y funcionalidad } \\
\text { de mi nariz }\end{array}$ & & & & \\
\hline
\end{tabular}

Tabla 2. Características generales de los grupos estudiados

\begin{tabular}{|lccc|}
\hline & $\begin{array}{c}\text { Grupo activo } \\
\text { Desean cirugía }\end{array}$ & $\begin{array}{c}\text { Control 1 } \\
\text { Hospitalizados }\end{array}$ & $\begin{array}{c}\text { Control 2 } \\
\text { Estudiantes }\end{array}$ \\
Edad (años) & $22,2 \pm 4$ & $51,5 \pm 15,2$ & $22,6 \pm 2$ \\
Escolaridad (años) & $14,8 \pm 2,1$ & $11,7 \pm 4,7$ & $16 \pm 0,9$ \\
IMC $\left(\mathrm{kg} / \mathrm{m}^{2}\right)$ & $22,92 \pm 3,5$ & $26,4 \pm 6,1$ & $22,9 \pm-2,4$ \\
n pacientes & 5 & 20 & 20 \\
\hline
\end{tabular}

Tabla 3. Mediana de respuestas por ítem en el preoperatorio

\begin{tabular}{|c|c|c|c|c|}
\hline & $\begin{array}{l}\text { Grupo activo } \\
\text { Desean cirugía }\end{array}$ & $\begin{array}{c}\text { Control } 1 \\
\text { Hospitalizados }\end{array}$ & $\begin{array}{c}\text { Control } 2 \\
\text { Estudiantes }\end{array}$ & P-value \\
\hline a) La apariencia de mi nariz es la mejor que puedo imaginar & 1 & 5 & 3,5 & 0,0037 \\
\hline b) Puedo respirar perfectamente por mi nariz & 5 & 5 & 5 & 0,4772 \\
\hline c) A mis amigos y familia les gusta mi nariz & 3 & 5 & 5 & 0,2767 \\
\hline $\begin{array}{l}\text { d) Nunca me he sentido limitado en mis actividades socia- } \\
\text { les-laborales por la apariencia de mi nariz }\end{array}$ & 4 & 5 & 5 & 0,0412 \\
\hline $\begin{array}{l}\text { e) Estoy seguro que la apariencia de mi nariz es la mejor } \\
\text { que puedo tener }\end{array}$ & 1 & 4 & 4 & 0,0037 \\
\hline $\begin{array}{l}\text { f) No me gustaría cambiar quirúrgicamente la apariencia y } \\
\text { funcionalidad de mi nariz }\end{array}$ & 1 & 5 & 5 & 0,0062 \\
\hline
\end{tabular}


Tabla 4. Resultado final de la encuesta por grupo de pacientes

\begin{tabular}{|lcc|}
\hline Grupo de pacientes & Nota en encuesta Preoperatorio & Nota en encuesta Postoperatorio \\
\hline Pcte. cirugía & 13,8 & 25,4 \\
Pcte. hospitalizado & 25,35 & 25,35 \\
Estudiante & 24 & 24 \\
\hline
\end{tabular}

\section{Discusión}

El desarrollo de investigación en cirugía plástica y el estudio de la calidad de vida en los pacientes operados, es uno de los desafíos actuales del área de investigación en salud. La Medicina Basada en Evidencia es fundamental para la producción de evidencia científica de calidad.

Para la realización del presente trabajo, comparamos las distribuciones en distintas poblaciones de pacientes sometidos a rinoplastía basados en un instrumento científicamente comprobado. Pero debemos aclarar que nuestros casos incluyen sólo pacientes sometidos a rinoplastía estética y no rinoplastía funcional. En el ítem "c" de la encuesta no obtenemos una diferencia estadísticamente significativa probablemente por la falta de tamaño en la muestra.

En relación a las falencias del trabajo original ${ }^{14}$, encontramos que la encuesta del Rhinoplasty Outcome Evaluation presenta afirmaciones dobles en los ítems "c, d y f" lo que resta especificidad a la respuesta Ej: familia-amigos, social-laboral, aparienciafuncionalidad.

\section{Conclusión}

Los resultados de nuestro estudio sugieren que la escala Rhinoplasty Outcome Evaluation posee validez de criterio adecuada y que es útil para evaluar la satisfacción con la apariencia nasal en nuestra población. Sin embargo, presenta falencias que son subsanables.

\section{Referencias}

1. American Society of Plastic Surgeons. 2012 Plastic Surgery Statistics Report. ASPS National Clearinghouse of Plastic Surgery Procedural Statistics 2012: 1-23. Disponible en www.plasticsurgery.org

2. Constantian M. Rhinoplasty. En: Craft \& Magic. St. Louis. Editorial Quality Medical Publishing Inc:
2009;1-1454.

3. Murphy M. Rhinoplasty. En: Master Techniques in Rhinoplasty. Philadelphia. Editorial Babak Azizzadeh 2011;1-500.

4. Sajjadian A, Naghshineh N, Rubinstein R. Current status of grafts and implants in rhinoplasty: Part II. Homologous grafts and allogenic implants. Plast Reconstr Surg. 2010;125:99-109.

5. Mazzola R, Felisati G. Rhinoplasty and Endoscopic Surgery for Functional and Inflammatory Nasal/Sinus Disorders. Plast Reconstr Surg. 2005;115:705-10.

6. Rohrich R, Janis J, Adams W, Krueger J. An Update on the Lateral Nasal Osteotomy in Rhinoplasty: An Anatomic Endoscopic Comparison of the External versus the Internal Approach. Plast Reconstr Surg. 2003;111:24612.

7. Pusic A, Klassen A, Phil D, Scott A, Klok J, Cordeiro P, et al. Development of a New Patient-Reported Outcome Measure for Breast Surgery: The BREAST-Q. Plast Reconstr Surg. 2009;124:345-53.

8. Klassen A, Cano S, Scott A, Snell L, Pusic A. Measuring Patient-Reported Outcomes in Facial Aesthetic Patients: Development of the FACE-Q. Plast Reconstr Surg. 2010;26:303-9.

9. Meningaud J, Lantieri L, Bertrand J. Rhinoplasty: An Outcome Research. Plast Reconstr Surg. 2008;121:2517.

10. McKiernan D, Banfield G, Kumar R, Hinton A. Patient benefit from functional and cosmetic rhinoplasty. Clin Otolaryngol Allied Sci. 2001;26:50-2.

11. Alsarraf R. Outcomes research in facial plastic surgery: A review and new directions. Aesthetic Plast. Surg. 2000;24:192-7.

12. Calderón M, Cuevas P, Erazo C, Benítez S, Andrades P, Sepúlveda S, y cols. Rinoplastía: Resultados Desde la Perspectiva del Paciente. Validación lingüística y psicométrica del rhinoplasty outcome evaluation instrument. Rev Chil Cir. 2013;65:30-4.

13. Likert R. A Technique for the Measurement of Attitudes". Archives of Psychology 1932;140:44-53.

14. Streiner D, Norman G. Health Measurement Scales. A Practical Guide to Their Development and Use. Ontario. Editorial Oxford Medical Publications 2003;15-27. 